\title{
Aptitud Combinatoria y Predicción de Híbridos de Maíz (Zea mays L.) de Grano Amarillo a Partir de Cruzas Dialélicas, Evaluadas en Dos Localidades de la Zona Baja de Guatemala, 19891
}

\author{
José Luis Quemé de León ${ }^{2}$, Luis Larios Bobadilla ${ }^{2}$, Carlos Pérez Rodas ${ }^{2}$ y Nery Soto León ${ }^{3}$
}

\section{COMPENDIO}

Por la importancia que tiene el maíz de grano amarillo en el crecimiento normal para humanos y animales y con el propósito de incrementar la producción de maíz en Guatemala, el Programa de Maíz del ICTA realizó la presente investigación utilizando 10 líneas en su mayoría con diferente origen genético y cuyo objetivo general planteado es generar cruzas dobles $(\mathrm{CD})$ y de tres líneas $(\mathrm{CT})$ que superen al mejor híbrido comercial HA-46; los objetivos específicos fueron: 1) Estimar los efectos de aptitud combinatoria general (ACG) de las líneas y aptitud combinatoria específica (ACE) de las cruzas simples (CS). 2) Realizar predicciones de rendimiento de grano de $\mathrm{CD}$ y $\mathrm{CT}$.

Se utilizó el diseño látice simple 7x7 para evaluar 42 CS mas de 7 testigos, para determinar la ACG y ACE se aplicó el diseño 4 de Griffing y para las predicciones se utilizó el método B de Jenkins. Se establecieron dos ensayos ubicados en los Centros de Producción de Cuyuta y La Máquina, los cuales están ubicados a 40 y al S0 msnm, respectivamente.

De acuerdo a los resultados obtenidos no se rechazaron las dos hipótesis planteadas por lo tanto: 1) La varianza de ACG $\left(\sigma_{\mathrm{g}}{ }^{2}\right)$ se considera igual a cero debido a que los progenitores fueron seleccionados previamente, mientras que la varianza de $\operatorname{ACE}\left(\sigma_{\mathrm{s}}^{2}\right)$ fue diferente de cero posiblemente porque algunos progenitores son de origen diferente mientras que otros provienen de la misma fuente. 2) Los efectos de ACE $\left(\mathrm{S}_{\mathrm{ijj}}\right.$ son tan importantes como los efectos de ACG $\left(\mathrm{g}_{\mathrm{i}}\right)$ en el rendimiento de las CS. Los rendimientos predichos de las CT y CD superaron al HA-46 predicho, por lo tanto, se espera que cuando estén formados los híbridos predichos, superen al HA-46 y con ello cumplir con el objetivo general trazado.

Palabras claves adicionales: hibridación, genotecnia, cruzas dohles, cruzas triples.

\section{INTRODUCCION}

El maíz es el alimento básico para los guatemaltecos, el cual aporta alrededor del45 \% de las calorías de la dieta diaria per capita y ocupa el primer lugar dentro de los granos básicos; este cereal se cultiva en un área de 619,200 ha, con un rendimiento de $1476 \mathrm{~kg} / \mathrm{ha}$ como promedio anual en el período de 1977 -19R2 (Ruano y Fumagalli, 1988).

El maíz de grano ,mlarillo juega un papel importante para humanos y animales, ya que según Jugenheimer, (1981) este maíz estimula un crecimiento normal, Ciertas áreas de la región IV de Guatemala, ubicadas en el litoral del Pacífico se han identificado por la producción de maíz amarillo; la semilla que utilizan los agricultores es en su ma-

\begin{abstract}
4
Given the importance that yellow grain maize hybrids have for human and animal consumption, the Maize Program of the Instituto de Ciencias y Tecnologia Agricola (ICTA) initiated a project using 10 maize lines to genera te double (DC) and three way cross (TWC) hybrids which would be superior to the commercial hybrid HA-46. Most of the 10 lines had different genetic origins. Specific objectives were: a) estimate specific and general combining ability (SCA, GCA) for each of the simple crosses; and b) to predict yield for the DC and TWC hybrids.
\end{abstract}

A $7 \times 7$ simple lattice design was used to evaluate 42 simple crosses and 7 checks. SCA and GCA were estimated using the Fourth Griffing's Design, and yield predictions using Jenkins Method B. Two trials were established in Cuyuta and La Maquina (40 to 150 meters above sea level).

Results obtained indicated that the two null hypothesis could not discarded: 1) the variance for $\mathrm{GCA}\left(\mathrm{g}^{2}\right)$ was considered to be equal to zcro given that the testers wcre previously selected; and the variance for SCA $\left({ }_{s}^{2}\right)$ was different from zero, possibly because some testcrs had different origins while others were derived from the same source. 2) SCA was as important as CGA in predicting the yicld of the simple crosses.

Predicted yield levels for DC's and TWC's were higher than the predicted yield for HA-46. These results suggest that when these DC's and TWC's are formed they will be superior to HA- 46 and as such fulfill the general objective set forth.

yoría variedades e híbridos generados por el Programa de Maíz del ICTA, citando por ejemplo la variedad A-6 y el híbrido HA-46.

Una opción para elevar la producción de maíz es el uso de híbridos de alto rendimiento, de aquí que el Programa de Maíz del ICTA le ha dado importancia a la generación de éstos, aplicando la metodología no convencional (híbridos de

${ }^{1}$ Trabajo presentado en la XXXVI Reunión Anual del PCCMCA, San Salvador, El Salvador, marzo 1990.

2 Técnico del Programa de Maíz, ICTA

${ }^{3}$ Coodirnador del Programa de Maíz, ICT A

${ }^{4} \mathrm{El}$ abstract es traducción del comprendio.

Publicado en Agronomía Mesoamericana, VoL 2 (1991) 
familias de hermanos completos y varietales) y convencional (de líneas).

La presente investigación está enfocada a la generación de hfbridos dobles (CD) y de tres líneas (CT), utilizando para ello materiales de diverso origen genético, con los cuales se pretente cumplir con los siguientes objetivos:

\section{General}

Generar cruzas dobles y de tres líneas de grano amarillo que superen al mejor híbrido comercial HA-46.

\section{Específicos}

Estimar los efectos de aptitud combinatoria general (ACG) de 10 líneas de grano amarillo y aptitud combinatoria específica (ACE) de las cruzas simples (CS).

Realizar predicciones de rendimiento de grano de CD y CT a partir de la información de las cruzas simples (CS).

\section{Hipótesis}

1. Dado que las líneas en estudio han sido seleccionadas previamente, la varianza ACG $\left(\sigma_{\mathrm{g}}{ }^{2}\right)$ se considera igual a cero, mientras que la varianza de ACE $\left(\sigma_{\mathrm{s}}^{2}\right)$ es diferente de cero debido a que algunos progenitores son de origen diferente, mientras que otros provienen de la misma fuente.

2. En el rendimiento de las CS, los efectos de ACE $\left(S_{i j}\right)$ serán importantes así como los efectos de ACG $\left(\mathrm{g}_{\mathrm{i}}\right)$ cuando menos en un progenitor.

\section{REVISIÓN DE LITERATURA}

La hibridación en maíz se considera como un método geno técnico que tiene como objetivo principal el aprovechamiento de la generación $\mathrm{F}_{1}$ (híbrido $\mathrm{F}_{1}$ ) provenientes del cruzamiento entre dos poblaciones $\left(\mathrm{P}_{1}\right.$ y $\left.\mathrm{P}_{2}\right)$ con cualquier estructura genotípica, las cuales pueden ser líneas endogámicas, variedades de polinización libre, variedades sintéticas o las poblaciones $\mathrm{F}_{1}$ mismas en el caso de las cruzas dobles (Márquez, 1988).

El valor de los progenitores de un híbrido se puede determinar mediante la prueba de ACG yACE. Sprague y Tatum (1942) definieron estos dos términos en relación al comportamiento relativo de las líneas al ser cruzadas; usando el vocablo aptitud combinatoria general para designar el comportamiento promedio de una línea en combinaciones híbridas, y aptitud combinatoria específica para designar a la desviación de cada combinación con respeto a la ACG de los progenitores; es decir, cada cruza puede ser mejor o peor de lo que se esperaría en base al comportamiento promedio de las líneas consideradas.

Brauer (1980) menciona que después de que se ha realizado la selección de líneas con base en su ACG (mediante cruzas probadoras o mestizos), sigue la prueba de ACE, la cual consiste en cruzar las líneas dos a dos en cruzamientos simples para ser evaluados en ensayos de rendimiento. Lo que se busca con esta prueba es identificar a los híbridos $F_{1}$ más rendidores de todas las cruzas simples posibles formadas a partir de líneas.

Griffing, citado por Martínez (1983), emplea la expresión cruzas dialélicas para describir un procedimiento en el cual se elige un conjunto de $\mathrm{P}$ líneas progenitoras y se realizan cruzas entre ellas; este mismo autor distingue cuatro diferentes técnicas de realizar dialélicas, las cuales varían dependiendo si se ensayan o no las autofecundaciones o'las cruzas recíprocas de las $F_{1}$.

Después de haber realizado la prueba de ACG y ACE, y teniendo los resultados de la evaluación de las CS provenientes de las líneas seleccionadas, la siguiente etapa es determinar las mejores combinaciones híbridas cuando se está interesado en CD y CT. Uno de los primeros investigadores en hacer estudios de predicción fue Jenkins (1934), quien propuso cuatro métodos para predicción del comportamiento de $\mathrm{CD}$ en maíz; por conveniencia, a cada método le asignó letras, A, B, C, y D, de acuerdo al estudio que realizó para rendimiento, el método $\mathrm{B}$ fue el que presentó mayor correlación (0.76); el método B utiliza para la predicción el promedio de las cuatro CS no paternas.

\section{MATERIALES Y MÉTODOS}

\section{Material genético}

Se ensayaron 42 cruzas simples provenientes entre las cruzas de 10 progenitores de diverso origen genético y diferente nivel de endogamia. Conjuntamente con las CS se evaluaron 7 testigos, los cuales se describen en el Cuadro 1.

\section{Localidades de evaluación de las cruzas simples}

Los ensayos se establecieron en los Centros de Producción del Instituto de Ciencia y Tecnología Agrícolas (ICTA), ubicados en las localidades de Cuyuta y la Máquina, las cuales se encuentran ubicadas en la zona tropical baja de Guatemala, a una altitud de 40 y $150 \mathrm{msnm}$. y con temperaturas promedio mensual de $27^{\circ} \mathrm{C}$. Los suelos de Cuyuta se caracterizan por ser franco-arenosos de la serie Tiquisate, con buen drenaje y los de La Máquina son franco-arcillosos, predominando los suelos de la serie Ixtán con mal drenaje.

\section{Período de evaluación}

La evaluación de los ensayos se realizó en el ciclo de 1989B, con siembras en la primera y tercera semana de junio para Cuyuta y La Máquina respectivamente, y la cosecha se realizó en la segunda semana de octubre para Cuyuta y la primera semana de noviembre para La Máquina. 
Cuadro 1 Descripción de las 10 líneas de maíz de grano amarillo que dieron origen a las cruzas dialélicas, la cuales se evaluaron con siete testigos en dos localidades de la zona baja de Guatemala. 1989.

\begin{tabular}{cll}
\hline Progenitores & Grado de Engoamia & \multicolumn{1}{c}{ Fuente } \\
\hline 1 & \multicolumn{1}{c}{$\mathrm{S}_{3}$} & Pool 21 \\
2 & $\mathrm{~S}_{3}$ & Pool 22 \\
3 & $\mathrm{~S}_{2}$ & \\
4 & $\mathrm{~S}_{3}$ & Pool 21 \\
5 & $\mathrm{~S}_{3}$ & Pool 21 \\
6 & $\mathrm{~S}_{3}$ & Población 28 \\
7 & $\mathrm{~S}_{5}$ & Población 24 \\
8 & $\mathrm{~S}_{6}$ & Población 27 \\
9 & $\mathrm{~S}_{6}$ & Población 27 \\
10 & $\mathrm{~S}_{3}$ & Pool 26 \\
& & \\
Testigos & & \\
HA-26 & Híbrido comercial de tres líneas & Pool 21 y Pob. 28 \\
Exp. 70 & Híbrido experimental de tres líneas & Pob. 28, Pool 26 y Pool 21 \\
Exp. 112 & Híbrido experimental de tres líneas & Pob. 28, Pool 26 y Pool 21 \\
X-3204 & Híbrido comercial & PIONEER \\
A-6 & Variedad de polinización libre & Pob. 28 \\
2207 (AL) & Híbridos resistentes a aluminio & Brasil \\
2211 (AL) & Híbridos resistentes a aluminio & Brasil \\
\hline
\end{tabular}

Nota: Las poblaciones y pooles han sido generados por CIMMYT.

Tamaño de la unidad experimental y distancia de siembra

La unidad experimental (parcela) estuvo constituída por dos surcos de $5 \mathrm{~m}$. de largo, separados a $75 \mathrm{~cm}$. entre surcos y $50 \mathrm{~cm}$ entre matas (dos plantas/mata), haciendo un total de 44 plantas por parcela $(53,333$ plantas/ha) siendo la parcela útil para los dos surcos.

\section{Toma de datos}

Se tomó el peso de mazorca de todas las plantas cosechadas en cada parcela, días a floración femenina, altura de planta, altura de mazorca, mazorcas descubiertas, aspecto de mazorca, tipo de grano y pudrición de mazorca.

\section{Análisis estadístico}

Se realizaron los análisis de varianza por localidad conforme a un diseño de látice simple, cuyo modelo matemático es el siguiente:

$$
Y_{i j k}=\mu+R_{k}+T_{i}+B_{j}\left(R_{k}\right)+\varepsilon_{i j k}
$$

donde:

$\mathrm{Y}_{\mathrm{ijk}}=$ valor observado en la unidad experimental

$\mu \quad=$ efecto medio

$\mathrm{R}_{\mathrm{k}} \quad=$ efecto de la repetición $\mathrm{k}$

$\mathrm{T}_{\mathrm{i}} \quad=$ efecto del tratamiento

$\mathrm{B}_{\mathrm{j}}\left(\mathrm{R}_{\mathrm{k}}\right)=$ efecto del bloque incompleto $\mathrm{j}$ de la repetición $\mathrm{k}$

$\varepsilon_{\mathrm{ijk}}=$ error asociado con la unidad experimental

\section{Análisis dialélico}

Para estimar los efectos de aptitud combinatoria se utilizó el diseño 4 de Griffing en bloques al azar, el cual se caracteriza por ensayar $\mathrm{p}$ ( $\mathrm{p}-1)$ / 2 combinaciones, donde $\mathrm{P}$ se refiere al número de progenitores. El modelo lineal para interpretar los resultados para este diseño es:

$$
\begin{gathered}
Y_{i j k}=\mu+g_{i}+g_{j}+S_{i j}+C_{i j k} \\
1 \leq \mathrm{i}, j \leq p, k=1,2 \ldots, r
\end{gathered}
$$

donde:

$\mathrm{Y}_{\mathrm{ijk}}=$ valor fenotípico observado de la cruza con progenitores i y j en el bloque $\mathrm{k}$

$\mu \quad=$ efecto común a todas las observaciones

$\mathrm{g}_{\mathrm{i}} \quad=$ efecto de la aptitud combinatoria general del progenitor $\mathrm{i}$

$\mathrm{S}_{\mathrm{ij}}=$ efecto de la aptitud combinatoria específica de la cruza $(i, j)$

$\mathrm{C}_{\mathrm{ijk}}=$ efecto ambiental aleatorio correspondiente a la observación (i, j, k)

Martínez (1983) menciona que para el diseño 4 de Griffing, los estimadores de la varianza del error $\left(\sigma_{\mathrm{e}}^{2}\right)$, de la ACG $\left(\sigma_{\mathrm{s}}^{2}\right)$ y de la ACE $\left.\sigma_{\mathrm{s}}{ }^{2}\right)$ se obtienen de los cuadrados medios (CM), de la siguiente manera:

$$
\begin{aligned}
\sigma_{\mathrm{e}}^{2} & =\mathrm{CM} \text { error } \\
\sigma_{\mathrm{s}}^{2} & =\frac{\mathrm{CM} \text { ACE }-\mathrm{CM}\{\text { error }\}}{\mathrm{r}} \\
\sigma_{\mathrm{g}}^{2} & =\frac{\mathrm{CM}\{\mathrm{ACG}\}-\mathrm{CM}\{\mathrm{ACE}\}}{\mathrm{r}(\mathrm{p}-2)}
\end{aligned}
$$

Según Velásquez (1978), las fórmulas para estimar " $\mu$ ", el efecto de ACG " $g_{\mathrm{i}}$ ” y el efecto de ACE " $\mathrm{S}_{\mathrm{ij}}$ ", son:

$$
\begin{aligned}
& \mu=\frac{2 Y}{r p(p-1)} \\
& g_{i}=\frac{Y_{i} . \cdot}{r(p-2)}-\frac{2 Y \ldots}{r p(p-2)} \\
& S_{i j}=\frac{Y_{i j}}{r}-\left(g_{i}+g_{j}\right)-Y_{. .}
\end{aligned}
$$


donde:

$\mathrm{r} \quad=$ número de repeticiones

$\mathrm{p} \quad=$ número de progenitores

$\mathrm{Y}_{\mathrm{i}}$. = suma de las cruzas en que interviene el progenitor $\mathrm{i}$

Y... = gran total

Predicción de híbridos: dobles y de tres líneas:

para la predicción del rendimiento de las CD se utilizó el método B de Jenkins, aplicando la siguiente ecuación:

$\mathrm{CD}_{\mathrm{ij} . \mathrm{kl}}=1 / 4\left(\mathrm{CS}_{\mathrm{ik}}+\mathrm{CS}_{\mathrm{il}}+\mathrm{CS}_{\mathrm{jk}}+\mathrm{CS}_{\mathrm{jl}}\right)$

donde:

$\mathrm{i}, \mathrm{j}, \mathrm{k}, 1$, representan a las cuatro líneas de una CD $(\mathrm{k} \times 1)$

$\mathrm{CD}_{\mathrm{ij} . \mathrm{kl}}=$ Valor de la predicción de la cruza doble $(\mathrm{i}$ x j)

$$
\begin{aligned}
\mathrm{CS}_{\mathrm{ik}}, \mathrm{CS}_{\mathrm{il}}, \mathrm{CS} \mathrm{jk} \text { y GS } \mathrm{jl}= & \begin{array}{l}
\text { comportamiento de las CS } \\
\text { no paternale o genotipos } \\
\text { predictores. }
\end{array}
\end{aligned}
$$

La predicción de las CT se realizó en forma semejante como se hizo en las cruzas dobles.

\section{RESULTADOS Y DISCUSIÓN}

En el Cuadro 2 se presentan el rendimiento de grano de las mejores $10 \mathrm{CS}$ consideradas sin diferencia significativa según el método de Tukey, en ambas localidades de evaluación. En la localidad de Cuyuta existieron rendimientos de las CS que superaron al híbrido HA-46 hasta en un 16\%, estas CS al igual que otras que no se incluyeron en el Cuadro 2 , presentaron los más altos rendimientos y sin diferencia significativa en la localidad de La Máquina, aunque los rendimientos en esta última localidad fueron bajos en comparación a la localidad de Cuyuta. Una explicación del bajo rendimiento en La Máquina se devió a la sequía que afectó en el período de floración de duró 30 días, y el valor acumulado de precipitación no llegó a los $20 \mathrm{~mm}$ y si a esto se le agrega la sensibilidad que tienen las CS a los cambios de ambiente, es logico que los rendimientos se redugeran drasticamente. Entre los testigos, el HA-46 fue uno de los mejores a pesar de que existió sequía, corroborando con ello el porqué este híbrido de tres líneas se haya recomendado para esta región. Los híbridos que presentaron los más bajos rendimientos fueron el 2207 (A1) y el 2211 (Al), atribuyendo estos resultados al problema de la sequía y a la inadaptación de los híbridos.

\section{Análisis de Varianza}

El resumen del análisis de varianza para la variable rendimiento de cada una de las dos localidades se presenta en el Cuadro No. 3. Existieron diferencias altamente significativas entre tratamientos, lo cual indica que por lo menos bay un tratamiento diferente a los demás; la media de rendimiento de Cuyuta (6.091 t / ha) fue tres veces superior a la media de La Máquina (2.037 t / ha), habiéndose explicado la causa de esta diferencia en el párrafo anterior, el coeficiente de variación para el análisis de Cuyuta se considera adecuado (8\%), no así el de La Máquina (20\%), el cual se puede deber a la desuniformidad en cuanto a la pendiente del terreno donde se estableció el experimento. En cuanto a la eficiencia relativa del diseño en látice con respecto al de bloques al azar (BCA), se puede decir que en la localidad de La Máquina, el diseño de látice sí fue más preciso que el BCA, no así para Cuyuta, en Por existir problemas de sequía en La Máquina y dado que los datos de los tratamientos para la variable rendimiento de esta localidad tienen similar tendencia a la de Cuyuta, se optó por hacer el análisis dialélico sólo para la localidad de Cuyuta.

Cuadro 2 Rendimiento de grano de las mejores cruzas simples

\begin{tabular}{|c|c|c|c|c|}
\hline \multirow[b]{2}{*}{ Cruza } & \multicolumn{2}{|c|}{ Cuyuta } & \multicolumn{2}{|c|}{ La Máquina } \\
\hline & t/ha* & \% sobre HA-46 & t/ha & $\%$ sobre HA-46 \\
\hline $7 \times 8$ & 7.864 & 116 & 2.650 & 101 \\
\hline $4 \times 9$ & 7.521 & 111 & 2.168 & 82 \\
\hline $4 \times 8$ & 7.260 & 107 & 2.792 & 106 \\
\hline $7 \times 9$ & 6.962 & 102 & 2.432 & 93 \\
\hline $2 \times 9$ & 6.886 & 101 & 3.568 & 136 \\
\hline $3 \times 4$ & 6.643 & 98 & 2.602 . & 99 \\
\hline $4 \times 6$ & 6.726 & 99 & 2.406 & 92 \\
\hline $2 \times 7$ & 6.639 & 98 & 3.242 & 123 \\
\hline $2 \times 4$ & 6.529 & 96 & 3.065 & 117 \\
\hline $2 \times 3$ & 6.185 & 91 & 3.216 & 122 \\
\hline \multicolumn{5}{|l|}{ Testigos } \\
\hline HA-46 & 6.793 & 100 & 2.628 & 100 \\
\hline Exp. 112 & 6.973 & 103 & 1.449 & 55 \\
\hline Exp. 70 & 6.536 & 96 & 2.082 & 79 \\
\hline A-6 & 5.510 & 81 & 2.025 & 77 \\
\hline$X-3204$ & 5.397 & 79 & 1.422 & 54 \\
\hline 2297 (AL) & 2.250 & 33 & 0.641 & 24 \\
\hline $2211(\mathrm{AL})$ & 2.005 & 30 & 0.386 & 15 \\
\hline $\bar{X}$ Gral. & 6.091 & & 2.037 & \\
\hline
\end{tabular}
consideradas sin diferencias significativa según el método de Tukey, en cada una de las localidades. 1989.

$* \mathrm{t} / \mathrm{ha}=$ toneladas métricas por hectárea

\section{Análisis de varianza del dialélico}

De acuerdo al análisis de varianza de las cruzas dialélicas (Cuadro 4), la ACG no fue significativa ( $\mathrm{P} \leq 0.05)$ mientras que la ACE fue altamente significativa $(\mathrm{P} \leq 0.01)$, indicando con ello que la selección ha sido más por su ACG que por su ACE (Márquez, 1988). La varianza de ACG $\left(\sigma_{\mathrm{g}}{ }^{2}\right)$ fue 
Cuadro 3 Resumen del análisis de varianza para la va riable rendimiento en las dos localidades de evaluación, 1989.

\begin{tabular}{lcccccc}
\hline & $\begin{array}{c}\text { Grados de } \\
\text { libertad }\end{array}$ & $\begin{array}{c}\text { Cuadrado Media } \\
\text { Medio }\end{array}$ & $\begin{array}{c}\text { CV } \\
(\mathrm{t} / \mathrm{ha})\end{array}$ & $\begin{array}{c}\text { E.R.L. } \\
(\%)\end{array}$ & $\begin{array}{c}\text { Intervalo } \\
\text { / }\end{array}$ \\
\hline Cuyuta & 48 & $2.927^{* * *}$ & 6.091 & 8 & 102 & $7.864-1.956$ \\
La Máquina & 48 & $0.969^{* * *}$ & 2.037 & 20 & 118 & $3.610-0.580$
\end{tabular}

$\mathrm{t} /$ ha $=$ toneladas métricas por hectárea

${ }^{2}$ E.R.L./BCA = Eficiencia relativa de látice con respecto a bloques completos al azar.

** $=$ Diferencia altamente significativa $(\mathrm{P} \leq 0.01)$
Cuadro 4 Análisis dialélico 'para rendimiento de las cruzas simples de 10 líneas de maíz de grano amarillo, evaluadas en la localidad de Cuyuta. 1989.

\begin{tabular}{|c|c|c|c|}
\hline $\begin{array}{l}\text { Fuente } \\
\text { Variación }\end{array}$ & $\begin{array}{c}\text { Grados de } \\
\text { libertad }\end{array}$ & $\begin{array}{l}\text { Suma de } \\
\text { Cuadrados }\end{array}$ & $\begin{array}{l}\text { Cuadrados } \\
\text { Medios }\end{array}$ \\
\hline Repeticiones & 1 & 1.226 & \\
\hline Cruzas & 41 & 70.184 & $1.712 * *$ \\
\hline $\mathrm{ACG}$ & 9 & 12.527 & $1.392 \mathrm{NS}$ \\
\hline $\mathrm{ACE}$ & 32 & 57.657 & $1.802 * *$ \\
\hline Error & 41 & 10.989 & 0.268 \\
\hline Total & 83 & 82.399 & \\
\hline
\end{tabular}

${ }^{1}$ Diseño 4 de Griffing en bloques al azar. Martínez (1983).

** Diferencia altamente significativa.

Cuadro 5 Efectos de ACG (gi) y ACE (Sij) en el rendimiento de cruzas dialélicas de maíz de grano amarillo, evaluadas en la localidad de Cuyuta, 1989.

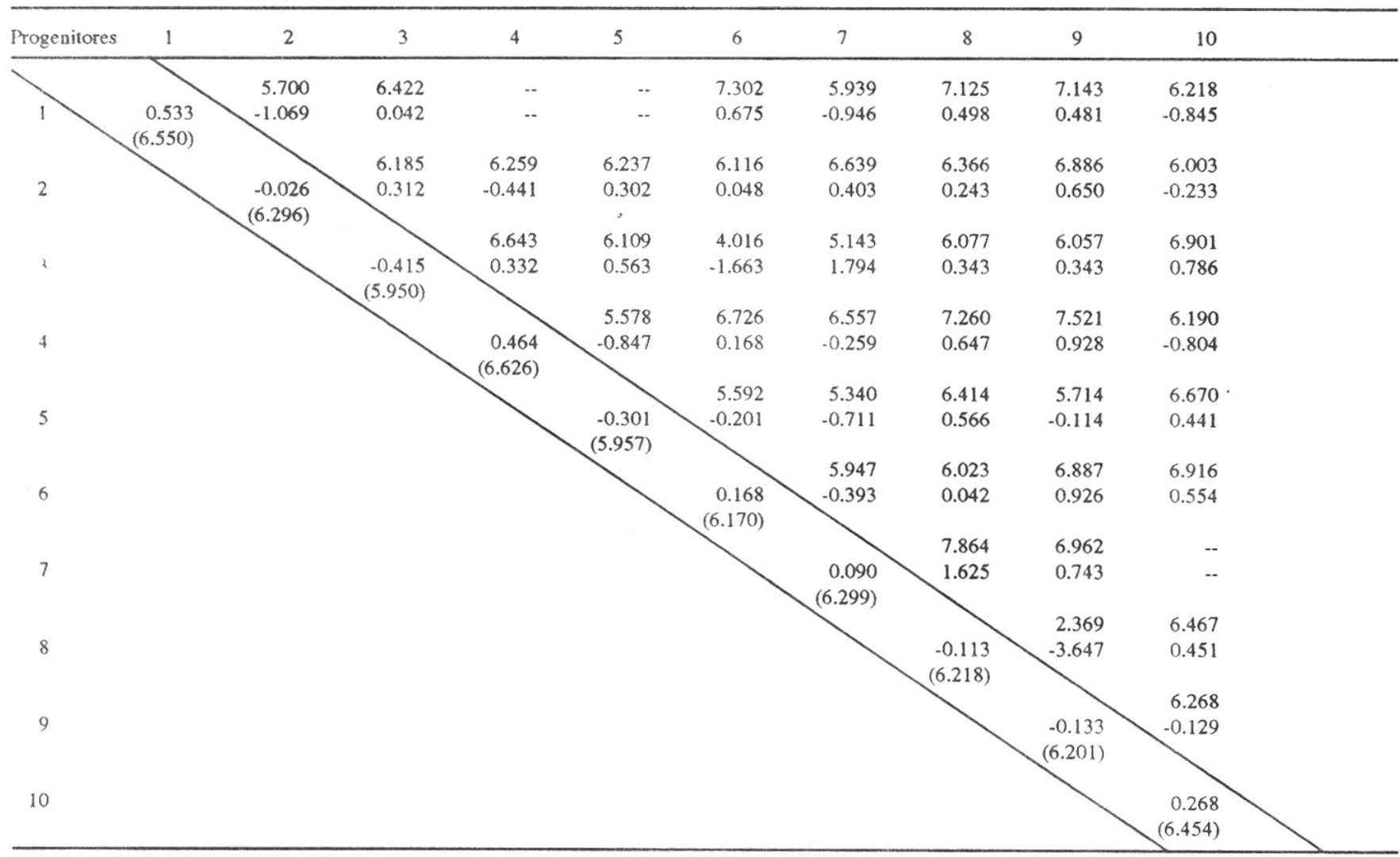

Diagonal principal: efectos de ACG; el número entre paréntesis es el promedio (en t/ ha) de las cruzas en que interviene cada progenitor.

Primera línea: rendimiento en $\mathrm{t} /$ ha de cada cruza.

Segunda línea: efectos de ACE de cada cruza.

de -0.025 , considerándose este valor como cero, mientras que la varianza de $\mathrm{ACE}\left(\sigma_{\mathrm{g}}{ }^{2}\right)$ fue de 0.767 . Estos resultados sirven de apoyo para no rechazar la hipótesis 1 , por lo que desde el punto de vista de ACG, las líneas se consideran similares, lo que hace suponer que la selección de las líneas ha sido efectiva para su ACG (Márquez, 1988). La importancia relativa de la $\sigma_{\mathrm{g}}{ }^{2}$ se pudo deber a que el dialélico estuvo constituido por cruzas emparentadas y no emparentadas, y según Velásquez (1978) indica que la varianza no aditiva adquiere más importancia en las cruzas entre proge- nitores no emparentados por la mayor expresión de efectos heteróticos.

Estos resultados coinciden con lo que menciona Sprague y Tatum (1942), que en material ya seleccionado por su ACG, la ACE es relativamente más importante que la ACG.

Efectos de $A C G\left(g_{i}\right)$ y ACE $\left(S_{i j}\right)$

De acuerdo al Cuadro 5, los progenitores 1, 4, 10 y 7 presentaron los valores más altos de ACG; sin embargo, ya 
Cuadro 6 Estructura genética de lasmejores 10 cruzas simples del dialélico de grano amarillo, evaluado en la localidad de Cuyuta, 1989.

\begin{tabular}{lccccc}
\hline Cruza & $\mathrm{Y}_{\mathrm{ij}}$ & $\mu$ & $\mathrm{g}_{\mathrm{i}}$ & $\mathrm{g}_{\mathrm{j}}$ & $\mathrm{S}_{\mathrm{ij}}$ \\
\hline $7 \times 8$ & 7.864 & 6.262 & 0.090 & -0.113 & 1.625 \\
$4 \times 9$ & 7.521 & 6.262 & 0.464 & -0.133 & 0.928 \\
$1 \times 6$ & 7.302 & 6.262 & 0.533 & -0.168 & 0.675 \\
$4 \times 8$ & 7.260 & 6.262 & 0.464 & -0.113 & 0.481 \\
$1 \times 9$ & 7.143 & 6.262 & 0.533 & -0.133 & 0.481 \\
$1 \times 8$ & 7.125 & 6.262 & 0.533 & -0.113 & 0.498 \\
$7 \times 9$ & 6.962 & 6.262 & 0.090 & -0.133 & 0.743 \\
$2 \times 9$ & 6.886 & 6.262 & -0.026 & -0.133 & 0.650 \\
$6 \times 9$ & 6.887 & 6.262 & -0.168 & -0.133 & 0.926 \\
$6 \times 10$ & 6.916 & 6.262 & -0.168 & -0.268 & 0.554 \\
\hline
\end{tabular}

$Y_{i j}, \mu, g_{i}, g_{j}$ y $S_{i j}$ : efectos del modelo matemático de Griffing.

Cuadro 7 Predicción del rendimiento de los mejores híbridos dobles y de tres líneas con base en la evaluación de cruzas dialélicas de maíz de grano amarillo en la localidad de Cuyuta, 1989.

\begin{tabular}{ccc}
\hline & Rend. Pred. & $\%$ Sobre \\
Híbrido Predicho & $(\mathrm{t} /$ ha $)$ & HA-46P
\end{tabular}

Dobles

$\begin{array}{lll}(4 \times 7)(8 \times 9) & 7.402 & 120 \\ (1 \times 7)(8 \times 9) & 7.274 & 118 \\ (1 \times 4)(8 \times 9) & 7.262 & 118 \\ (6 \times 9)(1 \times 4) & 7.173 & 116 \\ (2 \times 4)(8 \times 9) & 7.008 & 114\end{array}$

De Tres Líneas

$\begin{array}{lll}(4 \times 7) 8 & 7.562 & 123 \\ (1 \times 4) 9 & 7.332 & 119 \\ (1 \times 4) 8 & 7.193 & 117 \\ (1 \times 7) 9 & 7.053 & 115\end{array}$

Testigo

$\begin{array}{lll}(4 \times 5) 6=\text { HA-46P } & 6.159^{1 /} & 100 \\ \text { HA-46 } & 6.793^{2} & 110\end{array}$

${ }^{1}$ Se refiere al rendimiento del híbrido predicho del HA-46.

${ }^{2}$ Se refiere al rendimiento del híbrido HA-46 como tal.

se mencionó anteriormente que desde el punto de vista de ACG las líneas son similares; los g i guardan una relación estrecha con el rendimiento promedio de las cruzas en que interviene cada progenitor (en la diagonal entre paréntesis).

Los $\mathrm{S}_{\mathrm{ij}}$ para las $42 \mathrm{CS}$ varía desde 1.625 hasta - 1.663 . t/ha, esto era de esperarse ya que existió alta significancia para ACE. En el Cuadro 6, se presenta la estructura genética de las 10 mejores cruzas, se puede observar que en la mayoría de los casos en cada cruza, participa uno de los progenitores con mayor $\mathrm{g}_{\mathrm{i}}$, por lo tanto, se rechaza la hipótesis 2 , ya que el rendimiento de las CS son importantes tanto los $g_{i}$ como los $\mathrm{S}_{\mathrm{ij}}$.
Cuadro 8 Predicción del rendimiento de los mejores híbridos dobles y de tres líneas con base en la evaluación de cruzas dialélicas de maíz de grano amarillo en la localidad de La Máquina, 1989.

\begin{tabular}{ccc}
\hline Híbrido Predicho & $\begin{array}{c}\text { Rend. Pred. } \\
(\mathrm{t} / \mathrm{ha})\end{array}$ & $\begin{array}{c}\text { \% Sobre } \\
\text { HA-46P }\end{array}$ \\
\hline Dobles & & \\
$(2 \times 4)(9 \times 7)$ & 2.944 & 128 \\
$(2 \times 4)(7 \times 3)$ & 2.964 & 129 \\
$(2 \times 4)(8 \times 9)^{*}$ & 2.762 & 120 \\
$(4 \times 5)(7 \times 8)$ & 2.653 & 116 \\
$(4 \times 7)(8 \times 9)^{*}$ & 2.510 & 109 \\
$(1 \times 4)(8 \times 9)^{*}$ & 1.939 & 84 \\
De Tres Líneas & & \\
$(2 \times 3) 9$ & 3.104 & 135 \\
$(2 \times 4) 7$ & 3.020 & 132 \\
$(4 \times 7) 8 *$ & 2.721 & 119 \\
$(1 \times 4) 8 *$ & 2.078 & 91 \\
$(1 \times 4) 9 *$ & 1.799 & 78 \\
& & \\
Testigos & & 100 \\
$(4 \times 5) 6=$ HA-46P & $2.294^{1}$ & 115 \\
HA-46 & $2.628^{2}$ & \\
\hline
\end{tabular}

1 Se refiere al rendimiento del híbrido predicho del HA-46

${ }^{2}$ Se refiere al rendimiento del híbrido HA-46 como tal.

* Estos híbridos fueron también los mejores predichos para la localidad de Cuyuta.

\section{Predicción de CD y $C T$}

En el Cuadro 7 se presentan los rendimientos de las mejores 5 CD y 4 CT, las cuales superaron al híbrido HA-46 predicho hasta en un $23 \%$ en cuanto a rendimiento; estos datos se refieren a la localidad de Cuyuta.

En cuanto a la localidad de La Máquina, se realizaron 6 CD y 5 CT, las cuales superaron hasta en un 35\% al HA-46 predicho. Hay que hacer notar que de los híbridos predichos, 3 CD y 3 CT coincidieron en ambas localidades.

\section{CONCLUSIONES}

1. No se rechaza la hipótesis 1 , por lo tanto, la varianza de ACG $\left(\sigma_{\mathrm{g}}{ }^{2}\right)$ se considera igual a cero por haber utilizado líneas seleccionadas previamente, mientras que la varianza de ACE $\left(\sigma_{\mathrm{s}}^{2}\right)$ es diferente de cero posiblemente a que algunos progenitores provienen de igual o diferente fuente.

2. No se rechaza la hipótesis 2, concluyendo que los efectos de ACE $\left(\mathrm{S}_{\mathrm{ij}}\right)$ son tan importantes como los efectos de ACG $\left(g_{i}\right)$ en el rendimiento de las CS.

3. Las CD y CT predichas superaron al híbrido HA-46 predicho por lo que se espera que cuando estén formados los híbridos superen al HA-46 para cumplir con el objetivo general trazado. 


\section{BIBLIOGRAFÍA}

BRAUER H., 0.,1980. Fitogenética aplicada. Editorial Limusa, México p. 363-399.

JENKINS, M.T., 1934. Methods of estimating the performance of double in corn. J. Amer. Soc. Agron. 26:199-204.

JUSENHEIMER, R.A. 1981. Maíz., variedades mejoradas. medotdos de cultivos y producción de semillas. Primera edición. Editorial Limusa. México. P 525,

MARQUEZ S., F., 1988. Genotécnica Vegetal. Tomo II, A.C.T. Editor, S.A. México p.
MARTINEZ G.. A., 1983. Diseño y análisis de experimentos de cruzas dialélicas. Colegio de Postgraduados, Chapingo. México 252 p.

RUANO, S. y A. FUMAGALLI. 1988. Organización y manejo de la investigación en finca en el Instituto de Ciencia y Tecnología Agrícolas, ICTA Guatemala. 130 p.

SPRAGUE, G. F. and L. A. TATUM, 1942. General vs. specific cornbining ability in single crosses of corn. J.A. Soc. Agron. 34:923-932.

VELASQUEZ. M, R., 1978. Formación de híbridos simples en base a familias de hermanos completos, provenientes de diferentes poblaciones de maíz (Zea mays L.). Tesis de M.C., Colegio de Postgraduados. Chapingo, México. 84 p. 